\title{
Future roads for group-IV defect-enhanced quantum dot light-emitters for silicon photonics
}

\author{
Lukas Spindlberger ${ }^{1}$, Johannes Aberl ${ }^{1}$, Mark T. Lusk ${ }^{2}$, and Moritz Brehm ${ }^{1, *}$ \\ ${ }^{1}$ Semiconductor and Solid State Physics, Johannes Kepler University Linz, Altenberger Strasse 69, 4040 Linz, Austria \\ ${ }^{2}$ Department of Physics, Colorado School of Mines, Golden, Colorado 80401, United States \\ *email: moritz.brehm@jku.at, ORCID: 0000-0002-5629-5923
}

\begin{abstract}
Optical and optoelectronic properties of allgroup-IV quantum dot light-emitters for which the optical properties are enhanced by in-situ implantation of heavy ions (DEQDs) are investigated and discussed. Research directions towards more efficient light-emission from DEQDs as well as considerations towards the microstructural investigation of the nature of DEQD-defects are elaborated.
\end{abstract}

Keywords-Nanostructures, Low dimensional structures, Epitaxy, Ion-implantation, Germanium, Optoelectronics

\section{INTRODUCTION}

The intensified research efforts for efficient on-chip, group-IV-based light-emitters that preserve their optical properties well above room-temperature (RT) and that can be fabricated directly on Si are ongoing. Clearly essential for their CMOS-compatible integration are possibilities of electrical charge carrier injection and convenient coupling to other Si photonics components on standard SOI platforms, e.g. waveguides, detectors, etc.. Epitaxially-grown quantum dots (QDs) [1-4] offer benefits of charge carrier confinement and an all-crystalline matrix material, important for doping of e.g. light emitting diodes (LEDs) and laser diodes. A new class of all-group-IV light-emitters was discovered recently consisting of epitaxial Ge on Si QDs containing extended point defects, intentionally introduced upon low-energy ionimplantation [5-7]. In contrast to conventional Ge/Si QDs [24], for such defect-enhanced quantum dots (DEQDs), charge carriers are confined within the QD by two zero-dimensional but fundamentally different mechanisms [5,6]. Holes experience quantum confinement due to the large bandoffsets between $\mathrm{Ge}$ and $\mathrm{Si}$, while electrons are trapped by the introduced defects [6]. The most prominent features of DEQDs are clear signs for optically-pumped lasing [5,7], absence of thermal quenching of the photoluminescence (PL) emission up to RT [5-9], and the demonstration of LEDs working at $100^{\circ} \mathrm{C}$, grown directly on $\mathrm{Si}$ without the need of micrometer-thick buffer layers [9]. Nevertheless, in view of the intriguing but still poorly explored properties of these DEQD-based light-emitters and the perspectives of using them for Silicon-based photonics, strategies have to be developed towards (1) improvement of their light-emission efficiency, (2) understanding the microstructural and energetic nature of the defects-states upon their formation, robustness, optical properties, strain, etc. and (3) fostering the potential of DEQDs concerning devices in the field of optoelectronics and beyond.

\section{EXPERIMENTAL RESULTS AND MODELING}

\section{A. DEQD-fabrication procedure and optical properties}

In this work, Ge QDs are grown on $\mathrm{Si}(001)$ substrates bymolecular beam epitaxy by depositing $8 \AA$ of $\mathrm{Ge}$ at $500^{\circ} \mathrm{C}$ on a $45 \mathrm{~nm}$ thick crystalline Si buffer layer. For DEQD

We acknowledge funding by: Austrian Science Funds (FWF): P29137-N36 Linz Institute of Technology (LIT): LIT-2016-1-YOU-002 and QuantERA, co-funded under Horizon 2020, Grant Agreement No 731473. formation in-situ Ge ion-implantation was applied during the whole deposition time of Ge. The ion-implantation depth was matched to the QD height, i.e. $2 \mathrm{~nm}$, corresponding to $\mathrm{Ge}$ ion energies of $\sim 1.5 \mathrm{keV}$. For optical characterization, QD-overgrowth with crystalline $\mathrm{Si}$ is mandatory. A Si capping layer of $70 \mathrm{~nm}$ thickness was deposited at a growth temperature $T_{G}$ of $475^{\circ} \mathrm{C}$. Significantly lower and higher $T_{G}$ result into incomplete solid phase epitaxial regrowth and defect-complex migration out of the QD, respectively, both to be avoided by any means [6,7]. More details on the growth procedure can be found in [5-9].

In Fig. 1 the light-emission enhancement upon DEQD treatment is depicted by PL-spectra recorded at sample temperatures $\mathrm{T}_{\mathrm{S}}$ of $77 \mathrm{~K}, 222 \mathrm{~K}$ and $300 \mathrm{~K}$, respectively. At $\mathrm{T}_{\mathrm{S}}=77 \mathrm{~K}$ the DEQD sample (green) exhibit a $\sim 40$ times higher emission intensity as compared to reference QDs without Ge ion bombardment (black). This discrepancy increases for elevated $\mathrm{T}_{\mathrm{s}}$. While reference Ge/Si QDs do not emit light at RT, the emission intensity of DEQDs recorded at $300 \mathrm{~K}$ is more than 200 times higher than the one of reference $\mathrm{QDs}$ at $\mathrm{T}_{\mathrm{S}}=222 \mathrm{~K}$.

\section{B. Theoretical modeling}

First insights into the nature of the defect-structure of DEQDs were found in [6]. There, the minimum energy configuration of an amorphous Ge structure with one atom in excess of that for a perfect crystal of the same domain size upon annealing was calculated by density functional theory [5]. The atom reconfiguration upon annealing resulted in an extended defect structure known as a split-[110] interstitial which is surrounded by a neighborhood of $\sim 45$ displaced atoms. This defect-structure in the QD causes a downward shift of the lowest conduction band (CB) by $\sim 72 \mathrm{meV}$. It also results in a significant change in the $\mathrm{CB}$-alignment.

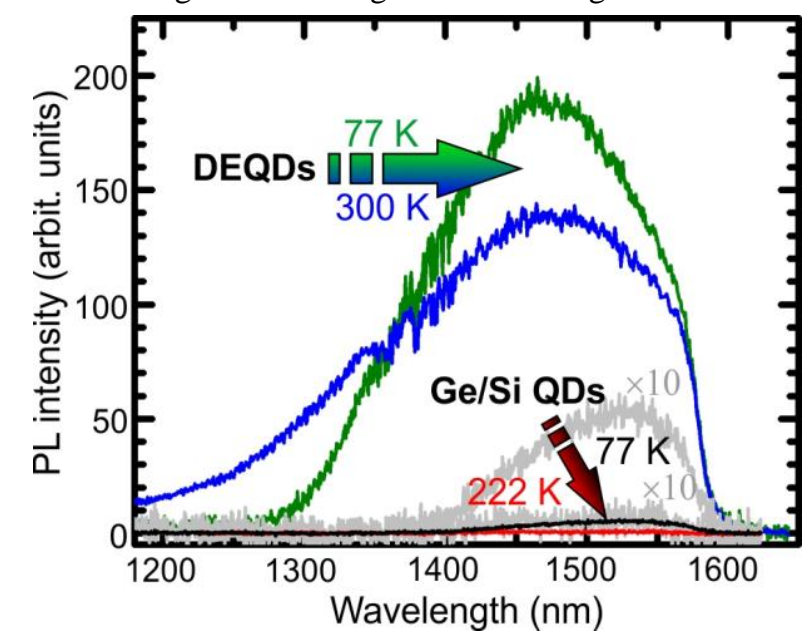

Fig. 1. Photoluminescence spectra of a DEQD-sample and a reference QD sample without additional $\mathrm{Ge}$ ion implantation. At $\mathrm{T}_{\mathrm{S}}=222 \mathrm{~K}$, a PLintensity increase of DEQDs as compared to unimplanted QDs can be observed. Gray spectra are magnified by a factor of 10 . 
In particular, the first band above the CB edge is localized at the defect at its $\Gamma$-point. For these calculations, the resulting calculated density of states (DOS) for DEQD-electron- and hole-states (magenta) are plotted with respect to the undisturbed Ge lattice (black) in Fig. 2. The original bandgap is indicated in yellow. Immediate conclusions from Fig. 2 are (1) that the DOS for electrons is increased (2) the bandgap is reduced as compared to crystalline $\mathrm{Ge}$ without split-[110] interstitial, and (3) hole-states are largely unaffected by the defect structure, albeit there still exist holestates within the bandgap of the nanostructure.

\section{DISCUSSION AND OUTLOOK}

Even though both, initial experimental and theoretical investigations were reported, overall the understanding of the DEQD platform, its optoelectronic properties, atomistic configuration, band structure and functionalization to devices is still at a rather basic level. Here, we want to draw a nonexclusive, coarse roadmap of what is necessary to advance the DEQD-platform.

\section{A. Enhancing the optical properties of DEQDs}

For any kind of optoelectronic application, the optical properties of DEQDs have to be advanced, e.g. lightemission from the single defect centers in QDs has to be optimized, non-radiative emission reduced, wave-function overlaps and optical matrix elements enhanced and the sheet density of active emission centers increased e.g. by vertical stacking of QDs [8]. Such goals can be targeted by changing the QD growth parameters to ensure formation of even smaller QDs, changes of the chemical composition of the QD and or matrix material to ensure optimum band alignment in the system and by changing the nature of the defect structure itself, see point IIIB. Furthermore, Hydrogen-treatment and advanced annealing techniques promise to reduce nonradiative recombination by saturation of unwanted defects created in the Si matrix. Thereby, the optical yield has to be monitored quantitatively, possibly by making use of integrating spheres and/or comparing DEQDs to emitters with well-known optical properties e.g. InAs/GaAs QDs [1].

\section{B. Defect alphabet and light-emission}

The split-[110] self-interstitial seems to be a minimum energy configuration for DEQDs considering implantation of Ge ions. Nonetheless, the question is valid if there exist other low-energy defect-configurations. This can become of particular importance considering the implantation of

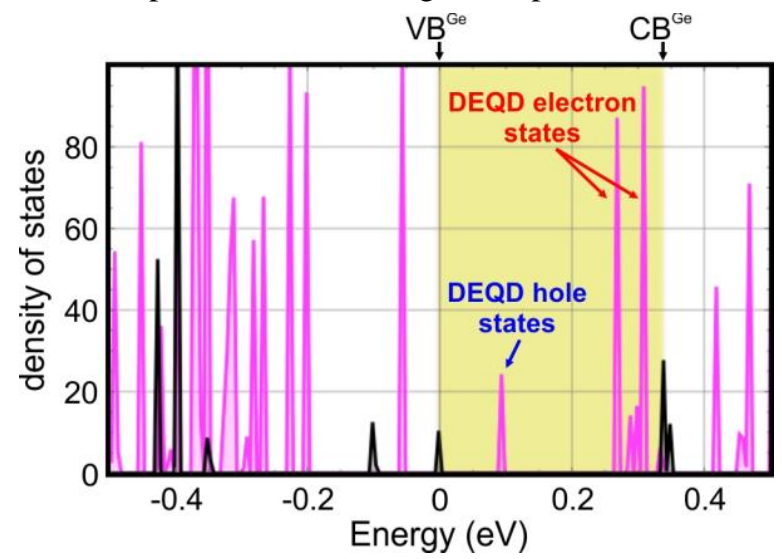

Fig. 2. Calculated DEQD electron- and hole-states (magenta) with respect to the undisturbed, relaxed Ge lattice (black). Note that DFT-results usually underestimate bandgap energies (here $0.34 \mathrm{eV}$ ) while energy differences of the states close to the band edges are rather accurately predicted. different ions than Ge for DEQDs. Although in previous studies only Ge ions were used for implantation, it is a priori not clear that a $\mathrm{Ge}$ ion should be the ideal candidate concerning the resulting optical properties of DEQDs. Also, the role of strain in the Ge QD crystal was not evaluated so far. The calculations in [6] were performed using a relaxed Ge crystal. In reality, it is, however, clear that the Ge film (QD) is pseudomorphically strained to almost $4 \%$ when deposited on $\mathrm{Si}(001)$. Thus, what happens to the defect configuration and the optical transitions if the strain status of the QD is changed? Experimentally this can be achieved either by changing the QD architecture [3], the growth of $\mathrm{SiGe}$ alloys on $\mathrm{Si}$, or deposition of $\mathrm{Ge}$ on a relaxed $\mathrm{SiGe}$ pseudo-substrate, etc.. Furthermore, the influence of potential hole-traps (Fig. 2) on the optical properties of DEQDs was not investigated so far. Can transitions involving defect-states for holes or, alternatively, the incorporation of heavier ions such as $\mathrm{Sn}$ or $\mathrm{Sb}$ into the QD contribute to a shift of the optical transitions further to the mid-infrared or $\mathrm{THz}$, regions that are highly important for sensing applications?

\section{DEQD-devices}

The realization of an electrically-driven DEQD-laser is a major milestone to be reached. However, the full potential of DEQDs has still to be unfolded. Applications towards DEQD-based detectors, sensors or other electronic or thermoelectric devices were not considered at all by now. Given the additional degree of freedom in the system, caused by the ion implantation into epitaxial QDs, we expect that the true potential of DEQDs as compared to fully crystalline QDs has yet to be unfolded.

\section{SUMMARY}

In this work, we have presented upcoming routes in the research of defect-enhanced quantum dots (DEQDs). The coexistence of crystalline regions and ones with disturbed crystallinity allows us to envision device applications beyond conventional optoelectronics, but also in other fields of electronics e.g. as thermoelectric coolers.

\section{ACKNOWLEDGMENT}

The authors thank M. Grydlik, T. Fromherz, P. Rauter, F. Schäffler and A. Rastelli for fruitful discussions.

\section{REFERENCES}

[1] K. Li, et al., J. Cryst. Growth, https://doi.org/10.1016/j.jcrysgro.2019.01.016, in print, (2019)

[2] G. Capellini, M. De Seta, L. Di Gaspare, F. Evangelisti, F. d'Acapito, J. Appl. Phys. 98, 124901 (2005).

[3] J. Zhang, M. Brehm, M. Grydlik, O. G. Schmidt, Chem. Soc. Rev. vol. 44 (1), pp. 26-39 (2015).

[4] L. Persichetti, A. Sgarlata, M. Fanfoni, A. Balzarotti, J. Phys. Condens. Matter, vol. 27, pp. 253001 (2015).

[5] M. Grydlik, F. Hackl, H. Groiss, M. Glaser, A. Halilovic, T. Fromherz, W. Jantsch, F. Schäffler, and M. Brehm, ACS Photonics, vol. 3 (2), pp. 298-303 (2016).

[6] M. Grydlik, M. T. Lusk, F. Hackl, A. Polimeni, T. Fromherz, W. Jantsch, F. Schäffler, and M. Brehm, Nano Lett., vol. 16 (11), pp. 6802-6807, (2016).

[7] M. Brehm and M. Grydlik, Nanotechn., vol. 28, pp. 392001 (2017).

[8] H. Groiss, L. Spindlberger, P. Oberhumer, F. Schäffler, T. Fromherz, M. Grydlik and M. Brehm, Semicond. Sci. Technol., vol. 32, pp. 02LT01, (2017).

[9] P. Rauter, L. Spindlberger, F. Schäffler, T. Fromherz, J. Freund, and M. Brehm, ACS Photonics, vol. 5, pp. 431-438 (2018) 